\title{
COMUNICAÇÃO BREVE Violência contra a mulher ou mulheres em situação de violência? Uma análise sobre a prevalência do fenômeno
}

\author{
Violence against women or women in a situation of violence? \\ An analysis on the prevalence of the phenomenon \\ Ricardo de Mattos Russo Rafael', Anna Tereza Miranda Soares de Moura²
}

\section{RESUMO}

Objetivo: Refletir sobre as possíveis diferenças entre os termos "violência contra mulher" e "mulheres em situação de violência" com base na análise da ocorrência de violência íntima. Métodos: Trata-se de um estudo transversal com uma amostra de 640 mulheres com idade entre 20 e 64 anos, em quatro unidades de Saúde da Família do município de Nova Iguaçu (RJ). Para aferição do evento, foi utilizada a versão em português do instrumento Revised Conflict Tactics Scales, que avaliou as formas - e suas interseções - de violência física, psicológica e sexual sofridas e perpetradas pelas mulheres ao menos uma vez na vida. Resultados: Estimou-se a prevalência das três formas de violência íntima praticadas contra a mulher (9,8\%; IC95\%: 7,5/12,1), pela mulher (4,5\%; IC95\%: 2,9/6.1) e nos casos que a mulher foi vítima ou perpetradora dos atos (13,1\%; IC95\%: 10,5/15,7), assumindo-se neste trabalho como violência no casal. Conclusão: $\mathrm{Na}$ tentativa de melhor entender a dinâmica

\section{Palavras-chave}

Violência, violência

doméstica, gênero e saúde. da violência íntima, seria oportuno considerar a mobilidade de papéis na relação do casal para além da demarcação de gênero - histórica e por vezes imobilizadora - entre vítima e agressor.

\section{ABSTRACT}

Objective: To reflect upon possible differences between the terms "violence against women" and "women in a situation of violence" from the analysis of the occurrence of intimate partner violence. Methods: It's a cross-sectional study with a sample of 640 women, aged between 20 and 64, in four Health Family Units in the municipality of Nova Iguaçu (RJ). The instrument used was the Portuguese version for the Revised Conflict Tactics Scales, that evaluated the forms - and their intersection - physical, psychological and sexual violence suffered and perpetrated by women at least once in life. Results: It has been estimated the prevalence of intimate forms of violence practiced against women (9.8\%; IC95\%: 7.5/12.1), by women (4.5\%; IC95\%: 2.9/6.1), and in the cases that the women was the victim or perpetrator of the acts (13.1\%; IC95\%: 10.5/15.7), it was assumed in this study as violence in couples.

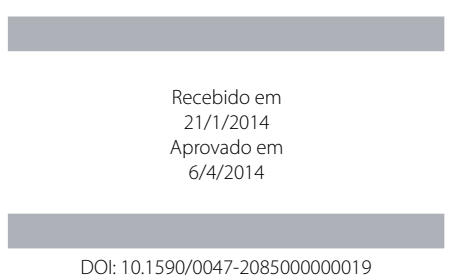

DOI: 10.1590/0047-2085000000019
1 Universidade do Estado do Rio de Janeiro (UERJ), Programa de Ciências Médicas; Universidade Iguaçu (Unig), Cursos de Graduação em Medicina e em Enfermagem.

2 UERJ, Ciências Médicas; Instituto de Medicina Social (IMS), Saúde Coletiva (Epidemiologia).

Endereço para correspondência: Ricardo de Mattos Russo Rafael

Av. Abílio Augusto Távora, 2134, bloco A, sala 203

26275-580 - Nova Iguaçu, RJ, Brasil

E-mail:prof.ricardomattos@gmail.com 


\section{Keywords}

Violence, domestic violence, gender and health.
Conclusion: In an attempt to better understand the dynamics of intimate partner violence, it would be useful to consider role mobility within the couple beyond the demarcation of genre - historical and sometimes immobilizing - between victim and assailant.

\section{INTRODUÇÃO}

Os eventos violentos vêm sendo compreendidos como fenômenos sócio-históricos de múltiplas causas e consequências. Apesar de sua complexidade, algumas questões parecem ter caminhado para melhor consenso, como a definição proposta pela Organização Mundial da Saúde e o seu modelo ecológico explicativo que tem servido como alicerce para o seu entendimento ${ }^{1}$. Especial destaque tem sido dado à violência entre parceiros íntimos, ganhando cada vez mais espaço nas agendas de discussão dos diversos setores da sociedade 2 . As consequências desses atos atingem, sobremaneira, as mulheres, gerando novos olhares no campo jurídico e criminal na tentativa de proteger as vítimas ${ }^{3,4}$.

A influência do gênero tem sido apontada como um dos mais significativos fatores entre aqueles que interferem na cadeia de causalidade da violência íntima, uma vez que reflete a visão social sobre os papéis dos homens e mulheres $^{1,4}$. Um dos gatilhos frequentes de atos violentos contra a mulher se refere a situações de contestação ou transgressão de regras tradicionalmente impostas pela sociedade, como a submissão e a dependência feminina'. A prevalência de agressões vivenciadas pelas mulheres, principalmente em sua forma psicológica, é alta, atingindo cerca de $80 \%$ em alguns estudos, e mesmo assim essas agressões ainda permanecem veladas 2,5 .

Por outro lado, embora existam estudos que se debrucem sobre a investigação da mulher como participante ativa nas situações que acabam por deflagrar os conflitos violentos entre o casal ${ }^{6,7}$, acredita-se que esse debate ainda careça de maior visibilidade social, principalmente no Brasil. Talvez pela influência da visão tradicional sobre as diferenças de gênero, em que a masculinidade pode ser colocada em risco quando o homem assume a posição de vítima, poucas são as informações referentes a esses atos violentos, quando comparados ao volume de trabalhos sobre a vitimização da mulher, o que dificulta a programação de estratégias para a compreensão do fenômeno. Além disso, os danos causados à saúde individual e à qualidade das relações íntimas podem ocorrer mesmo e também quando a mulher assume a posição de perpetradora.

O presente artigo traz um recorte de um projeto maior que avalia as barreiras no acesso do rastreio do câncer do colo uterino considerando as relações da violência íntima e o uso abusivo de álcool em usuárias da Saúde da Família e tem por objetivo refletir sobre as diferenças dos termos "violência contra mulher" e "mulheres em situação de violência" com base na análise da ocorrência de violência entre o casal.

\section{MÉTODOS}

Foi realizado um estudo seccional em quatro unidades de Saúde da Família, totalizando 11 equipes da região centro de Nova Iguaçu, Rio de Janeiro. Na amostragem aleatória de mulheres atendidas no período de novembro de 2012 a junho de 2013, foram captadas 680 respondentes, das quais 640 voluntárias responderam às entrevistas em um ambiente privado nas dependências das unidades. Em todos os casos as mulheres foram abordadas por entrevistadoras previamente treinadas e sem a companhia do parceiro, a fim de garantir privacidade e segurança a elas. Consideraram-se como critérios de inclusão estar na faixa etária de 20 a 64 anos, ter parceiro íntimo há pelo menos um ano e ser residente na área de cobertura da estratégia.

O instrumento utilizado na coleta de dados foi composto por questões de caracterização sociodemográfica da amostra e pelo Revised Conflict Tactics Scales (CTS2) ${ }^{8}$, considerando a gravidade dos atos violentos e as posições da mulher como vítima e perpetradora dos eventos (física, psicológica e sexual) com seus parceiros íntimos ao longo da vida. Os dados foram tratados no programa Stata 12 SE (Stata Corp., College Station, Estados Unidos) com análise descritiva, sendo calculados prevalências e respectivos intervalos de confiança (IC 95\%), considerando-se caso de violência quando as mulheres respondentes referiram ao menos um item de cada escala como positivo. A violência no casal foi considerada positiva na presença de atos violentos quando a muIher estava na posição de vítima e/ou perpetradora.

O projeto foi submetido ao Comitê de Ética, com autorização sob o CAAE 01724512.6.0000.5259, e considerou as recomendações da Organização Mundial de Saúde sobre pesquisas envolvendo as situações de violência9. Além disso, todas as participantes assinaram os Termos de Consentimento Livre e Esclarecido e, quando necessário, foram encaminhadas à rede de suporte do município.

\section{RESULTADOS}

Não houve perdas ao longo da coleta de dados, com percentual de recusas de 3\%. A tabela 1 apresenta a caracterização sociodemográfica da amostra. O predomínio foi de mulheres na faixa etária de 30 a 49 anos (50,4\%; IC95\%: 46,5/54,3), pretas e pardas (56,1\%; IC95\%: 52,3/59,9), casadas (55,5\%; IC95\%: $51,6 / 59,3)$, com tempo de estudo até oito anos $(57,8 \%$; IC95\%: 54,0/61,6) e pertencentes à classe socioeconômica C (70,7\%; IC95\%: 67,2/74,3). 
Tabela 1. Perfil sociodemográfico da amostra de mulheres residentes na área de cobertura da Estratégia de Saúde da Família, Nova Iguaçu, 2013

\begin{tabular}{|c|c|}
\hline Características da amostra $(n=640)$ & Prevalência \% (IC95) \\
\hline \multicolumn{2}{|l|}{ Faixa etária } \\
\hline$<30$ anos & $14,0(11,3 / 16,7)$ \\
\hline 30-39 anos & $26,1(22,7 / 29,6)$ \\
\hline 40-49 anos & $24,2(20,9 / 27,6)$ \\
\hline 50-59 anos & $27,2(23,8 / 30,7)$ \\
\hline 60 anos e mais & $8,3(6,2 / 10,5)$ \\
\hline \multicolumn{2}{|l|}{ Raça/Cor } \\
\hline Branca & $40,6(36,8 / 44,4)$ \\
\hline Preta & $23,9(20,6 / 27,2)$ \\
\hline Parda & $32,2(28,5 / 35,8)$ \\
\hline Amarela/indígena & $3,3(1,9 / 4,7)$ \\
\hline \multicolumn{2}{|l|}{ Tempo de estudo } \\
\hline Até 8 anos & $57,8(54,0 / 61,6)$ \\
\hline Mais de 8 anos & $42,2(38,3 / 46,0)$ \\
\hline \multicolumn{2}{|l|}{ Situação conjugal } \\
\hline Casada & $55,5(51,6 / 59,3)$ \\
\hline Outros & $44,5(40,7 / 48,4)$ \\
\hline \multicolumn{2}{|l|}{ Classe econômica segundo ABEP* } \\
\hline$A / B$ & $20,2(17,0 / 23,3)$ \\
\hline c & $70,7(67,2 / 74,3)$ \\
\hline $\mathrm{D} / \mathrm{E}$ & $9,1(6,8 / 11,3)$ \\
\hline
\end{tabular}

*ABEP: Associação Brasileira de Empresas de Pesquisa.

A tipologia das violências é demonstrada por sexo, no casal e estratificada por gravidade na tabela 2. As figuras 1 e 2 apresentam as interseções dos diferentes tipos de violência investigados quando a mulher era vítima ou perpetradora, bem como ocorrendo entre o casal.

Tabela 2. Prevalência de violência íntima segundo vitimização em usuários da Estratégia de Saúde da Família, Nova Iguaçu, RJ, $2013(n=640)$

\begin{tabular}{lccc}
\hline Tipologia & Vítima & Perpetradora & Casal \\
\hline Violência física & $21,0(17,9 / 24,3)$ & $22,6(19,4 / 25,9)$ & $28,6(25,1 / 32,1)$ \\
Grave & $5,9(4,0 / 7,6)$ & $5,8(4,0 / 7,6)$ & $8,4(6,3 / 10,6)$ \\
Menor & $19,7(16,6 / 22,8)$ & $21,1(17,9 / 24,3)$ & $26,6(23,1 / 30,0)$ \\
Violência psicológica & $90,7(88,5 / 93,0)$ & $91,8(89,7 / 94,0)$ & $92,2(90,1 / 94,3)$ \\
Grave & $67,6(64,0 / 71,3)$ & $71,1(67,6 / 74,6)$ & $75,1(71,8 / 78,5)$ \\
Menor & $90,0(87,7 / 92,3)$ & $91,1(88,9 / 93,3)$ & $91,2(89,0 / 93,4)$ \\
Violência sexual & $39,1(36,0 / 43,6)$ & $14,3(11,9 / 17,4)$ & $39,9(36,6 / 44,3)$ \\
Grave & $6,6(4,6 / 8,5)$ & $0,07(0,009 / 1,4)$ & $6,7(4,7 / 8,6)$ \\
Menor & $38,4(34,6 / 42,2)$ & $14,5(11,8 / 17,3)$ & $38,9(35,1 / 42,7)$ \\
\hline
\end{tabular}
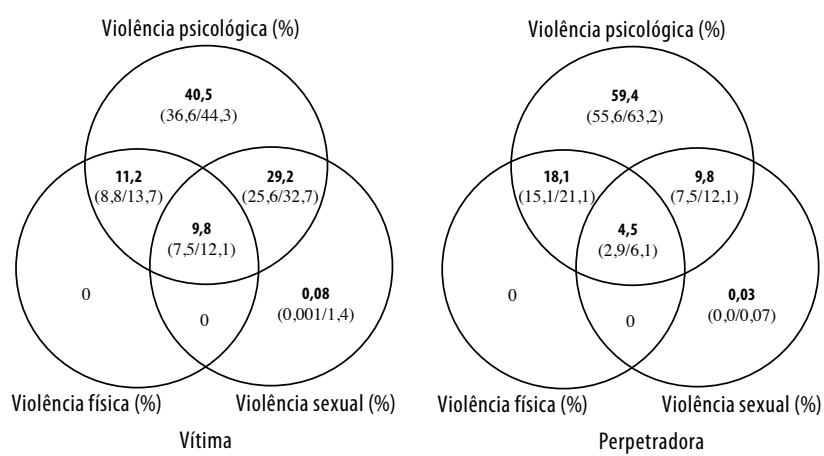

Figura 1. Interseções entre os tipos de violência íntima \% (IC95\%) segundo posição de vítima e perpetradora, em usuárias da Estratégia de Saúde da Família do município de Nova Iguaçu, RJ, 2013 ( $n=640)$.

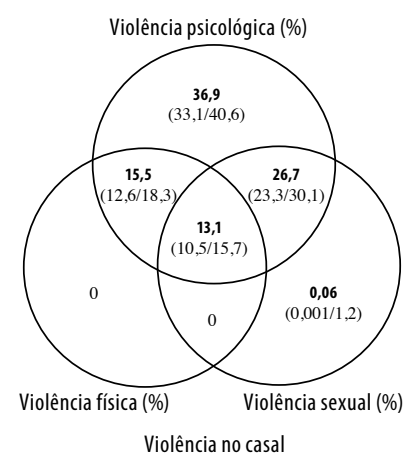

Figura 2. Interseções entre os tipos de violência íntima \% (IC95\%) em usuárias da Estratégia de Saúde da Família do município de Nova Iguaçu, RJ, 2013 ( $n=640$ ).

\section{DISCUSSÃO}

Os resultados apresentados possibilitam uma visão mais detalhada da violência entre o casal, ao assinalar as diferenças - ou similaridades - entre as prevalências encontradas no papel da mulher como vítima e perpetradora, respectivamente. Eles possibilitaram observar que a ocorrência dos atos violentos perpetrados pelo casal parece estar se equilibrando, excetuando-se a violência sexual, quando comparados a resultados obtidos no início desta década ${ }^{7,10}$. A redução da violência física como forma isolada de agressão também é ponto de destaque, passando a ocorrer principalmente em concomitância às agressões psicológicas.

Essa possível diferença de práticas violentas perpetradas contra a mulher pode ter várias explicações, como os aspectos judiciais sobre o tema e amplas campanhas na mídia que revelam a elevada ocorrência e graves consequências à saúde feminina. O empoderamento das mulheres sobre os seus direitos, a rigorosa legislação atual e a modificação na composição dos núcleos familiares parecem contribuir para as mudanças atualmente experimentadas na sociedade ${ }^{3}$. Parece que uma nova atitude social está sendo tomada relativa- 
mente ao abuso físico, com redução de sua aceitação como forma de manifestação do poder masculino. No entanto, percebe-se que a ocorrência de outras formas de violência permanecem elevadas, principalmente aquelas que podem ser ocultadas 5 . Existem situações mais íntimas em que a submissão feminina ainda está presente, como as relações sexuais nas quais as diferenças de gênero são fortemente dominantes. Apesar dos avanços, a mulher ainda permanece desqualificada em suas relações, com elevada frequência de abusos psicológicos, talvez na tentativa de velar as marcas deixadas por outras formas de ameaças dos parceiros.

A violência perpetrada contra o homem ainda é marginalizada no âmbito doméstico, reforçando-se quase sempre a posição de vítima da mulher nesse espaço. Os resultados mostram que a quase totalidade dos homens do estudo foi exposta a algum tipo de abuso. Com a exceção dos abusos sexuais, os homens são vitimados quase na mesma proporção das mulheres. Analisando-se as inter-relações entre as formas violentas, percebe-se que a mulher também agride e, na maior parte dos casos, de forma psicológica. Da mesma forma que já existe a compreensão de que a violência pode se apresentar com a justaposição de suas diferentes tipologias, percebe-se que existe certa dinâmica no papel de vítima e perpetrador(a) ${ }^{7}$. Essa não é uma assunção nova, mas pode descortinar algo que ainda permanece no campo do proibido pela ótica masculina - a vitimização de abusos perpetrados por mulheres ${ }^{10}$.

Não se pretende com essa inferência retroceder historicamente e reforçar o papel da mulher como principal responsável pelos conflitos domiciliares ou reforçar as noções tradicionais de gênero que traduziam a submissão do feminino; pretende-se justamente o contrário. Os resultados do estudo apontam para a necessidade de manter um olhar sobre as relações conflituosas abusivas do casal, sugerindo que as raízes e as consequências também são compartilhadas. A limitação sobre um único foco pode ampliar, de forma cíclica e progressiva, o grau de sofrimento para os sujeitos envolvidos ${ }^{11}$.

E é nesse cenário que a compreensão da codependência do casal, amplamente utilizada na abordagem do uso e abuso de substâncias químicas, pode ser uma contribuição significativa para o trabalho com as situações de violência entre parceiros íntimos. Entende-se a codependência como um conjunto de condutas que produzam um sofrimento psíquico, ou mesmo físico, para todos os atores envolvidos direta ou indiretamente com o ato violento ${ }^{12}$. Nesse sentido, encarar os abusos como situacionais parece mais prudente do que o emprego de uma nomenclatura voltada à culpa e à vitimização.

Parece importante considerar as limitações de estudos seccionais presentes neste trabalho, principalmente pelo fato de as mulheres terem sido as responsáveis pelas in- formações referentes à ocorrência da violência em ambos os sexos. Na tentativa de minimizar possíveis vieses, foram adotadas as recomendações referentes à manutenção de ambientes privativos e seguros, bem como a aplicação das entrevistas por mulheres ${ }^{13}$.

É possível perceber que o emprego do termo "violência contra a mulher" pode trazer uma visão limitada sobre o fenômeno por não contemplar as suas muitas outras facetas. A mudança na terminologia para "situações de violência" - já empregada por diversas instituições envolvidas com o tema - não é meramente estilística, e sim semântica, garantindo o emprego de estratégias voltadas à abordagem sistêmica e contextual. A mudança nos papéis entre vítima e perpetrador(a) da violência já é temática conhecida e defendida na literatura atual10, entretanto a investigação sobre a forma de compreensão dessas relações ainda é meta a ser alcançada nos contextos que envolvem parceiros íntimos.

\section{CONCLUSÃO}

A constante vitimização da mulher pela sociedade, mídia e investigações científicas pode estar gerando perdas de componentes necessários para a compreensão do fenômeno. Dessa forma, o estudo pretendeu iluminar de maneira diferenciada uma parte do problema, enfatizando a necessidade de contemplar a dinâmica de cada casal, contrapondo as especificidades dos gêneros para o manejo desse tipo de violência.

\section{CONTRIBUIÇÕES INDIVIDUAIS}

Ricardo de Mattos Russo Rafael - Trabalhou na concepção e desenho do estudo, na análise e interpretação dos dados, na elaboração e revisão crítica do conteúdo, bem como na aprovação da versão final a ser publicada.

Anna Tereza Miranda Soares de Moura - Trabalhou na concepção e desenho do estudo, na análise e interpretação dos dados, na elaboração e revisão crítica do conteúdo, bem como na aprovação da versão final a ser publicada.

\section{CONFLITOS DE INTERESSE}

Não há conflitos de interesse.

\section{AGRADECIMENTOS}

Agradecemos à Fundação de Amparo à Pesquisa do Estado do Rio de Janeiro (Faperj) pelo financiamento deste estudo. 


\section{REFERÊNCIAS}

1. Krug EG, Dahlberg LL, Mercy JA, Zwi A, Lozano R, editores. Relatório mundial sobre violência e saúde. Genebra: Organização Mundial da Saúde; 2002.

2. Moura LBA, Gandolfi L, Vasconcelos AMN, Pratesi R. Violências contra mulheres por parceiro íntimo em área urbana economicamente vulnerável, Brasília, DF. Rev Saud Publica. 2009;43(6):944-53.

3. Medrado B, Méllo RP. Posicionamentos críticos e éticos sobre a violência contra as muIheres. Psicol Soci. 2008;20(especial):78-86

4. Minayo MCS. Violência e saúde. Rio de Janeiro: Fiocruz; 2006

5. Schraiber LB, Barros CRS, Castilho EA. Violência contra as mulheres por parceiros íntimos: usos de serviços de saúde. Rev Bras Epidemiol. 2010;13(2):237-45.

6. Whitaker DJ, Haileyesus T, Swahn M, Saltzman LS. Differences in frequency of violence and reported injury between relationships with reciprocal and nonreciprocal intimate partner violence. Am J Public Health. 2007;97(5):941-7.

7. Reichenheim ME, Szklo A, Hasselmann MH, Souza ER, Lozana JA, Figueiredo V. The magnitude of intimate partner violence in Brazil: portraits from 15 capital cities and the Federal District. Cad Saude Publica. 2006;22(2):425-37.
8. Moraes CL, Hasselmann MH, Reichenheim ME. Adaptação transcultural para o português do instrumento "Revised Conflict Tactics Scales (CTS2)" utilizado para identificar violência entre casais. Cad Saude Publica. 2002;18(1):163-76.

9. World Health Organization. Putting women first: ethical and safety recommendations for research on domestic violence against women. Geneva: WHO; 2001.

10. Drijber BC, Reijnders UJL, Ceelen M. Male victims of domestic violence. J Fam Viol. 2013;28:173-8

11. Acosta F. Conversas homem a homem: grupo reflexivo de gênero. Rio de Janeiro: Instituto Noos; 2004

12. Moraes LMP. Atenção de enfermagem ao familiar do dependente químico: grupo como estratégia do cuidar [tese]. Fortaleza (CE): Universidade Federal do Ceará - Programa de Pós-Graduação em Enfermagem; 2008.

13. Rafael RMR, Moura ATMS. Considerações éticas sobre pesquisas com mulheres em situações de violência. Rev Bras Enferm. 2013;66(2):287-90. 